\title{
Protective Effects of Almond Oil on Streptozotocin-Induced Diabetic Rats via Regulating Nrf2/HO-1 Pathway and Gut Microbiota
}

\author{
Rui Liu (D), Ying Shu (D), Wenhui Qi $\left(\mathbb{D}\right.$, Weili Rao ${ }^{(D)}$, Zihan Fu $(\mathbb{D}$, Zhenxiao Shi $(\mathbb{D}$, \\ and Zhisheng Zhang $\mathbb{C}$ \\ College of Food Science and Technology, Hebei Agricultural University, Lekai South Avenue, Baoding, Hebei 071000, China \\ Correspondence should be addressed to Zhisheng Zhang; zhangzhisheng66@139.com
}

Received 27 January 2021; Revised 23 April 2021; Accepted 11 May 2021; Published 7 June 2021

Academic Editor: Biao Yuan

Copyright (C) 2021 Rui Liu et al. This is an open access article distributed under the Creative Commons Attribution License, which permits unrestricted use, distribution, and reproduction in any medium, provided the original work is properly cited.

\begin{abstract}
Almond oil has been used as a medicine substitution for its numerous health benefits. This study aimed to evaluate the effect of almond oil on streptozotocin- (STZ-) induced diabetic rats for 4 weeks. The results showed that the administration of almond oil could significantly increase body weight, attenuate abnormally elevated blood glucose, promote insulin secretion, and improve glucose tolerance. Almond oil treatment also suppressed oxidative stress, reduced inflammation reaction, improved liver and kidney function, upregulated the expressions of Nrf2, HO-1, and NQO1, while downregulating the expression of Keap1. Furthermore, almond oil reversed the gut microbiota change by STZ and regulated the gut microbiota associated with glucose metabolism. At the phylum level, the relative abundance of Firmicutes was decreased, while Bacteroidetes was increased by almond oil treatment. More importantly, the ratio of Firmicutes/Bacteroidetes was significantly increased. At the genus level, administration of almond oil increased the abundances of Lactobacillus, Bacteroides, and Lachnospiraceae_NK4A136_group, while decreased the abundances of Ruminococcaceae_UCG-014, Clostridium_sensu_stricto_1, and Fusicatenibacter. These results provided evidence for the regulating effect of almond oil on diabetic rats via the Nrf2/HO-1 pathway and gut microbiota.
\end{abstract}

\section{Introduction}

Diabetes is a chronic metabolic disease characterized by hyperglycemia that has been recognized as an increasing global health problem [1]. Genetics and environments are important factors in the pathogenesis of diabetes. In 2017, approximately 451 million adults lived with diabetes, and the number was estimated to rise to 693 million by 2045 throughout the world [2]. Chronic hyperglycemia causes oxidative stress and inflammation in the occurrence of diabetes. Besides, it is frequently associated with dysfunction and severe clinical complications, such as diabetic retinopathy [3], nephropathy [4], neuropathy [5], and peripheral artery disease, owing to the glucotoxicity effects $[6,7]$, which accelerate the mortality of diabetes. Therefore, controlling a high level of blood glucose and alleviating the generation of reactive oxygen species (ROS) are essential for controlling diabetes or ameliorating diabetic complications.

The nuclear factor erythroid 2-related factor $2(\mathrm{Nrf} 2)$ is an intracellular transcription factor that could maintain cellular redox homeostasis and upregulate cytoprotective proteins, such as heme oxygenase $1(\mathrm{HO}-1)$ and $\mathrm{NAD}(\mathrm{P}) \mathrm{H}$ quinone oxidoreductase 1 (NQO1). Under normal conditions, the binding of Kelch-like ECH-associated protein 1 (Keap1) to Nrf2 could suppress the Keap1/Nrf2 pathway, which leads to the ubiquitination and degradation of cytoplasmic Nrf2 [8]. The deficiency of expression of Nrf2 could enhance the susceptibility to many oxidative stress-related pathologies [9]. Some agents could protect diabetes-related organs or cells from injuries by upregulating the expression of Nrf2 [10]. Nrf2 regulates the expressions of a series of antioxidant enzymes and is considered an effective target on oxidative stress- 
related diseases [11]. Heme oxygenase-1 (HO-1) is a downstream cytokine of $\mathrm{Nrf2}$, which exerts a vital function in maintaining the redox balance of cells [12]. The Nrf2/HO-1 pathway plays a critical role in regulating oxidative stress.

More importantly, the gut microbiota plays a critical role in host homeostasis, and the dysbiosis of the gut microbiota is strongly associated with the onset and progress of metabolic diseases, such as diabetes $[13,14]$. A previous study found that commensal bacteria, such as Lactobacillus plantarum, can promote the transfer of insulin vesicles and insulin secretion by ligand binding with NOD1 in islet $\beta$ cells [15]. Furthermore, a decreased proportion of anaerobes, especially Bacteroides, can lead to hyperglycemia. The aberrant microbiota in STZinduced diabetic rats and the upsurge of the Gram-negative bacteria could lead to inflammation and the development of a pathological microenvironment [16]. Lipopolysaccharide (LPS) as a gut-derived endotoxin might be crucially involved in chronic inflammation. Release of LPS by pathogenic bacteria in the intestine can enter the bloodstream and trigger low-level inflammation and oxidative damage in rats with high intestinal permeability. Reducing the levels of LPSproducing bacteria in diabetic rats might contribute to reducing systemic inflammation and promoting normal liver insulin signaling [17]. Therefore, regulation of the gut microflora may be beneficial for reversing the inflammatory and maintaining the balance of glucose metabolism. Diet is an important factor altering the composition and metabolism of the gut microbiota, especially the main dietary macronutrients whose amount, type, and balance greatly impact the large intestinal microbiota [18].

Some natural products isolated from plant sources had therapeutic and antioxidant properties and could be used as a form of supplement or replacement with antioxidant activity and fewer side effects, which should be searched for attenuating the risk of diabetes. Almond (Prunus amygdalus) is the most popular nuts and widely used as ingredients in some processed foods such as bakery and confectionery products. A previous study showed that the consumption of almonds could reduce the risk of chronic diseases and prevent colon cancer [19]. Besides, almond contains proteins, certain minerals, vitamins E and D, and about 50\% oil. Almond oil has high amounts of monounsaturated fatty acids (MUFA), especially a rich concentration of oleic fatty acids [20], which could alleviate cellular apoptosis, oxidative stress, mitochondrial dysfunction, and inflammation in hepatocytes [21]. Almond oil has long been used as a medicine substitution [22] and has significant potential in biomedical and pharmacological studies. Previous studies illustrated that the consumption of almond oil could inhibit lipid peroxidation processes in $\mathrm{CCl}_{4}$-induced hepatic damage rats and boost immunity $[23,24]$ due to its anti-inflammatory and antioxidant activity and free radical scavenging capacity. However, the gut microbiota changes in STZ-induced diabetes by the dietary supplement of almond oil were hardly reported.

Due to the excellent antioxidant activity of almond oil, we speculated the beneficial effect of almond oil on diabetes. Therefore, our present study aimed to investigate the effects of almond oil on diabetic rats induced by STZ via the regulation of oxidative stress and inflammatory response,
Nrf2/HO-1 signaling pathway, and gut microbiota changes. The study would provide a beneficial suggestion for choosing a diet supplement.

\section{Materials and Methods}

2.1. Chemicals. The almond oil was kindly provided by Laiyuan Almond Processing Co., Ltd., in Hebei, China, in November 2019. The sunflower seed oil was purchased from a local grocery store in Baoding, Hebei, China, in November 2019 , as the positive control. They were both stored at $4^{\circ} \mathrm{C}$ before use.

Streptozotocin (STZ) was purchased from SigmaAldrich Co. (St. Louis, MO, USA).

The biochemical kits of superoxide dismutase (SOD), catalase (CAT), malondialdehyde (MDA), alanine transaminase (ALT), aspartate transaminase (AST), creatinine (CRE), and blood urea nitrogen (BUN) were obtained from Jiancheng Bioengineering Research Institute (Nanjing, China). The enzyme-linked immunosorbent assay (ELISA) kits of inflammation indicators of tumor necrosis factor $\alpha$ (TNF- $\alpha$ ) and interleukin $1 \beta$ (IL-1 $\beta$ ), insulin (INS), and advanced glycation end products (AGEs) were purchased from Boster Biological Technology Co., Ltd. (Wuhan, China).

2.2. Composition Analysis of Almond Oil. The composition analysis of almond oil was detailed in Table S1.

2.3. Animals and Experimental Treatment. Male Sprague Dawley rats (200 220 g) were purchased from Beijing HFK Bioscience Co., Ltd. The rats were kept under standardized conditions at a constant temperature of $23-25^{\circ} \mathrm{C}$, and $50 \pm 5 \%$ humidity with a $12 \mathrm{~h}$ light/dark cycle, and they had free access to standard diet and water for one week adjustment period. All experimental procedures and animal welfare were performed under a protocol that was approved by the Research Ethics Committee of the College of Food Science and Technology in Hebei Agricultural University.

After the adjustment period, the rats were weighed and randomly divided into six groups $(n=10)$ : the control group $(\mathrm{CON})$, the model control group $(\mathrm{MC})$, the low dose of almond oil group (LAO), medium dose of almond oil group (MAO), high dose of almond oil group (HAO), and sunflower seed oil group (SSO). All rats were fasted for $12 \mathrm{~h}$, and rats in $\mathrm{MC}, \mathrm{LAO}, \mathrm{MAO}, \mathrm{HAO}$, and SSO were intraperitoneally injected STZ with $60 \mathrm{mg} / \mathrm{kg}$, which was dissolved in $0.1 \mathrm{M}$ citrate buffer ( $\mathrm{pH} 4.2-4.5$ ), while those in $\mathrm{CON}$ were intraperitoneally injected with the same dose of citrate buffer solution. Fasting blood glucose (FBG) was determined three days after the injection. The rats with levels of FBG $>16.7 \mathrm{mmol} / \mathrm{L}$ were considered as diabetic rats for experiments.

Rats in LAO, MAO, and HAO were intragastrically administrated with almond oil at doses of 2,4 , and $8 \mathrm{~g} / \mathrm{kg}$ b.w. and SSO administrated with sunflower seed oil at a dose of $4 \mathrm{~g} / \mathrm{kg}$ b.w. for 4 continuous weeks, respectively, while rats in CON and MC were given at an equal volume of sterile water.

Body weights and FBG of all the rats were monitored weekly throughout the whole experimental period. At the 
last week of the experiment, oral glucose tolerance test (OGTT) was determined. At the end of the experiment, the rats were deprived of food overnight and $10 \%$ chloralic hydras $(6 \mathrm{ml} / \mathrm{kg})$ was intraperitoneally injected for anesthesia. The blood samples were collected from the abdominal artery aorta of rats and separated by centrifugation at $3000 \mathrm{rpm}$ for $15 \mathrm{~min}$ to obtain serum and then stored at $-80^{\circ} \mathrm{C}$ for further assay. The liver samples were rinsed with saline and dried on filter paper, then quickly snap-frozen, and preserved at $-80^{\circ} \mathrm{C}$ for Western blotting analysis.

The colon contents sample of each rat was collected at a sterile condition and preserved at $-80^{\circ} \mathrm{C}$ for further microbiota analysis.

2.4. Measurement of Fasting Glucose and OGTT. FBG levels were measured from the tail vein of rats after overnight $(12 \mathrm{~h})$ fasting using a glucose meter. The OGTT was estimated at the last week after administration of AO or SSO. The blood glucose levels were estimated at $0,30,60$, and 120 minutes after administration with glucose solution $(2 \mathrm{~g} / \mathrm{kg}$ $\mathrm{BW}$ ). The area under the curve (AUC) during the OGTT was calculated by the trapezoid rule.

2.5. Biochemical Parameters Analysis. The enzyme activities of SOD, CAT, MDA, ALT, AST, CRE, and BUN in the serum were determined by biochemical kits; INS, TNF- $\alpha$, IL- $1 \beta$, and AGEs in the serum were quantified by ELISA kits strictly according to the instructions of instruments and reagents.

2.6. Western Blot Analysis. The expressions of Keap1, Nrf2, HO-1, and NQO1 protein in liver were determined by Western blotting. The liver samples were homogenized and lysed in lysis buffer containing a protease inhibitor on ice. The suspension was centrifuged at $10,000 \mathrm{~g}$ for $10 \mathrm{~min}$ at $4^{\circ} \mathrm{C}$ and the supernatant was collected for Western blot assay. The total protein concentration was quantified using a BCA assay kit. Equal concentrations of protein samples were separated by $10 \%$ SDS-polyacrylamide gel electrophoresis (PAGE) and then transferred to the polyvinylidene fluoride membranes (PVDF). The membranes were blocked with 5\% BSA in TBST buffer for $1 \mathrm{~h}$ and incubated in primary antibodies against Keap1, Nrf2 $(1: 1000)$, HO-1, NQO1 $(1: 10000)$, and $\beta$-actin $(1: 4000)$ at $4^{\circ} \mathrm{C}$ overnight. The membranes were washed 5 times in TBST and incubated with HRP-conjugated antirabbit or anti-mouse secondary antibodies at room temperature for $1 \mathrm{~h}$. After washing 7 times with TBST, the protein bands were visualized by enhanced chemiluminescence reagent (Thermo, Rockford, USA). Finally, bands were analyzed using image analysis system (Bio-Rad, CA, USA).

2.7. Fecal DNA Extraction and Sequencing. According to the manufacturer's instructions, total DNA was extracted from colon contents using the TIANamp Stool DNA kit (Tiangen, Beijing, China). The bacterial 16S rRNA gene V3-V4 region was amplified with primers 338F ( $5^{\prime}$-ACTCCTACGGGAGGCAGCAG-3 $\left.{ }^{\prime}\right)$ and 806R (5'-GGACTACHVGGGTWTCTAAT- $\left.3^{\prime}\right)$. The PCR amplification program was performed as follows: initial denaturation at $95^{\circ} \mathrm{C}$ for $3 \mathrm{~min}$, followed by 27 cycles PCR $\left(95^{\circ} \mathrm{C}\right.$ for $30 \mathrm{~s}$, $55^{\circ} \mathrm{C}$ for $30 \mathrm{~s}$, and $72^{\circ} \mathrm{C}$ for $30 \mathrm{~s}$ ), finally $72^{\circ} \mathrm{C}$ for $10 \mathrm{~min}$. The PCR product was extracted from $2 \%$ agarose gel, purified using AxyPrepDNA (Axygen Biosciences, Union City, CA, USA), quantified using Quantus ${ }^{\mathrm{TM}}$ Fluorometer (Promega, USA), and finally sequenced using Illumina MiSeq platform (Illumina San Diego, USA) at Majorbio Bio-Pharm Technology company (Shanghai, China).

Sequences with $\geq 97 \%$ similarity were assigned to the same operational taxonomic units (OTUs). The sequences were trimmed with UCHIME and annotated using RDP Classifier (http://rdp.cme.msu.edu/) based on the SILVA database (SSU123) at the threshold of 70\%.

2.8. Statistical Analysis. Data were expressed as mean \pm SD. Statistical analysis was performed using SPSS 17.0. Statistical significance between groups was analyzed using one-way analysis of variance (ANOVA) followed by Duncan's new multiple range test. $P$ values $<0.05$ or $P$ values $<0.01$ were considered statistically significant or statistically highly significant.

\section{Results}

3.1. Effect of Almond Oil on Body Weight in Diabetic Rats. The changes in body weight are presented in Figure 1. Body weight of the rats decreased significantly one week after the injection of STZ $(P<0.01)$. The body weight among diabetic groups had no significant differences during the first two weeks. However, treatment with almond oil could obviously reverse the reduction during 4-week administration. Compared with the MC group, the body weight of the MAO group was significantly increased at week $2(P<0.05)$; the three doses of almond oil and the SSO group significantly increased body weight $(P<0.01)$ at weeks 3 and 4 . The results showed that different doses of almond oil could reduce the weight loss of diabetic rats.

\subsection{Effect of Almond Oil on FBG, OGTT, and Insulin Levels in} Diabetic Rats. Elevated FBG is one of the main characteristics of diabetes. The FBG was estimated every week. As shown in Figure 2(a), FBG of the MC group was significantly higher than the CON group after STZ induction $(P<0.01)$, which proved that the diabetes model had been established. Compared with the MC group, there was no significant difference in FBG in rats during the first two weeks' intervention. However, FBG of the LAO and HAO groups exhibited a significant descent tendency $(P<0.05)$ compared with the MC group at week 3, and FBG was decreased by $20.9 \%$ in the LAO group at the last week. The results suggested that $\mathrm{AO}$ could suppress the increase in FBG.

OGTT aims to measure the ability to tolerate glucose in rats. Results of OGTT among all groups are shown in Figure 2(b). The maximum blood glucose level occurred 30 minutes after the oral administration of glucose, and the MC group had a significantly lower level of glucose tolerance compared with the CON group $(P<0.01)$. The LAO group 


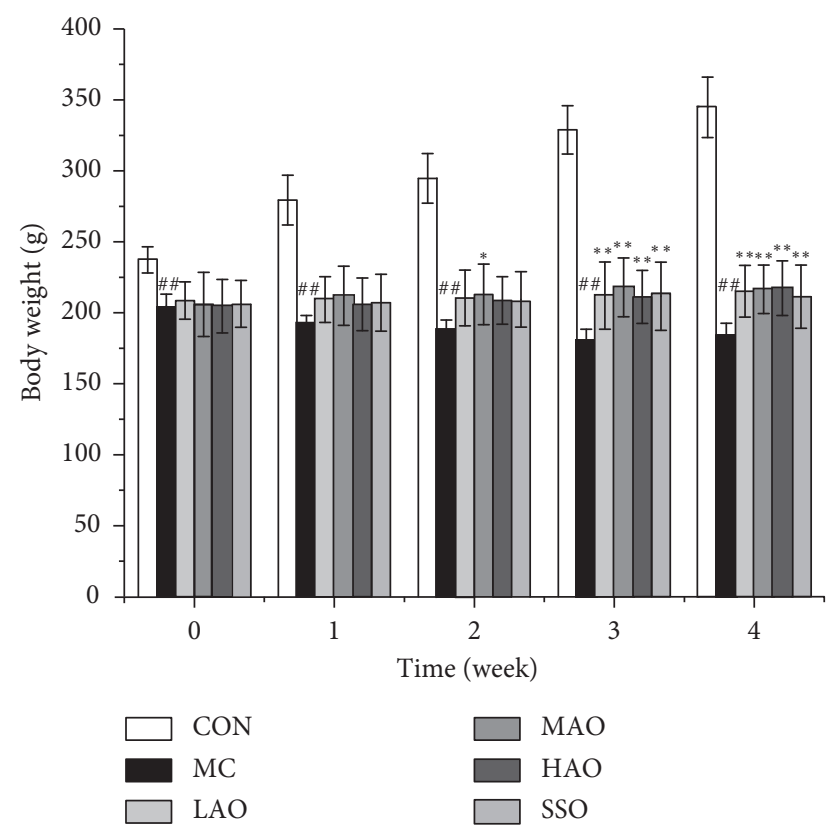

FIGURE 1: Effect of almond oil on body weight in STZ-induced diabetic rats. CON, control group; MC, model control group; LAO, low dose of almond oil-treated group; MAO, middle dose of almond oil-treated group; HAO, high dose of almond oil-treated group; SSO, sunflower seed oil-treated group. Data were expressed as means \pm SD. The MC group compared with the CON group, ${ }^{\#} P<0.05,{ }^{\# \#} P<0.01$; the LAO, $\mathrm{MAO}, \mathrm{HAO}$, and SSO groups compared with the MC group, ${ }^{*} \mathrm{P}<0.05,{ }^{* *} P<0.01$.

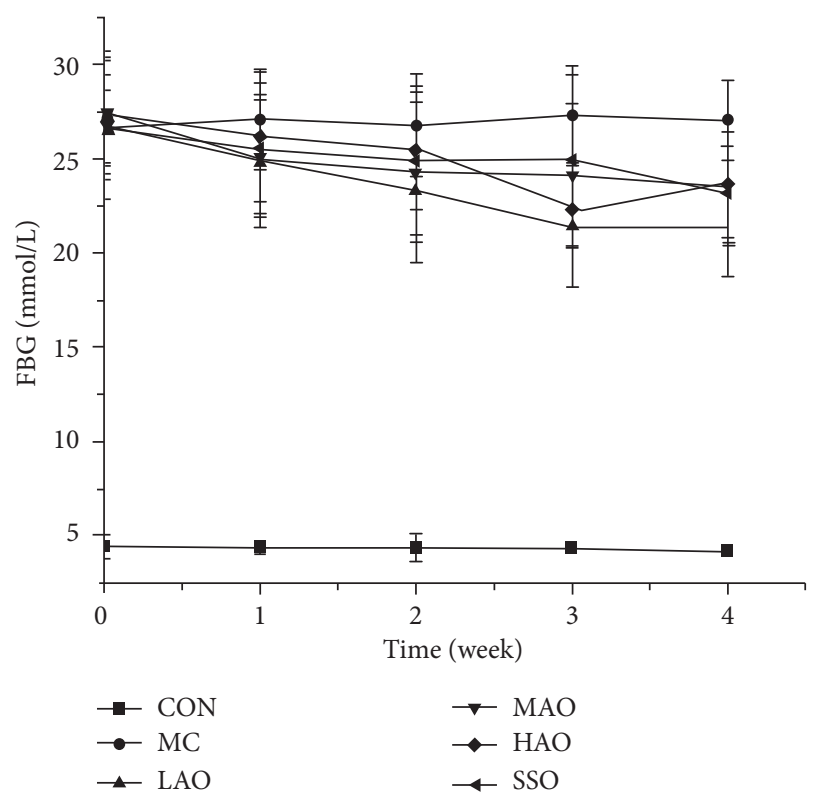

(a)

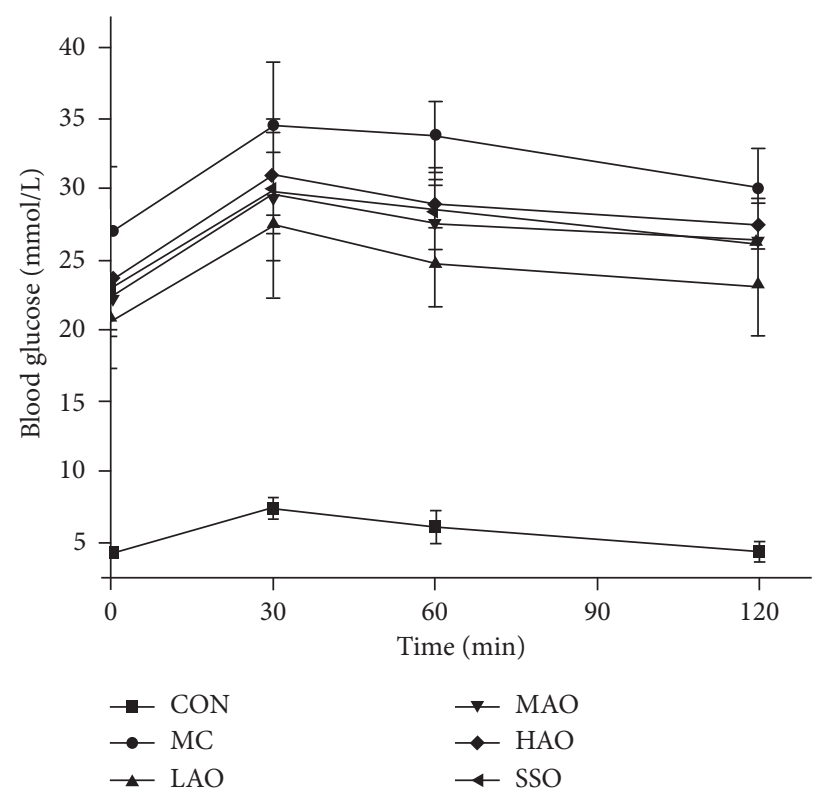

(b)

FIGURE 2: Continued. 


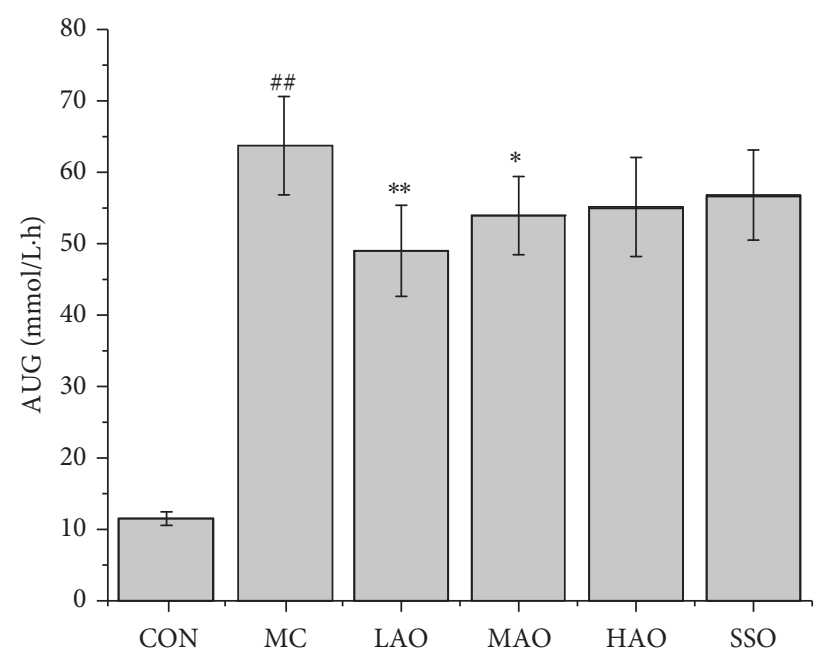

(c)

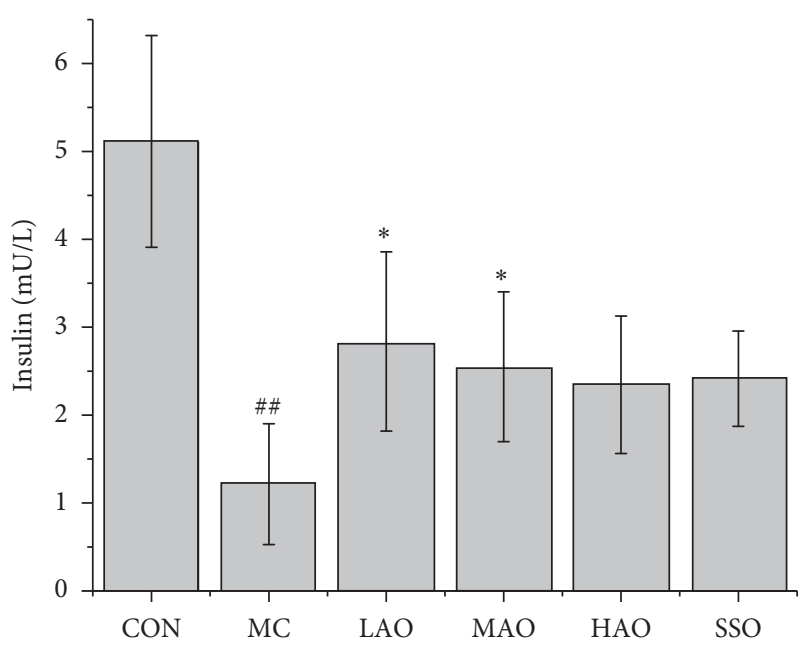

(d)

Figure 2: Effects of almond oil on the FBG, glucose tolerance, and insulin levels in STZ-induced rats. (a) Fasting blood glucose. (b) Glucose tolerance. (c) Area under the curve of blood glucose (AUC). (d) Serum insulin. Data were expressed as means \pm SD. The MC group compared with the CON group, ${ }^{\#} P<0.01,{ }^{\# \#} P<0.01$; the LAO, MAO, HAO, and SSO groups compared with the MC group, ${ }^{*} P<0.05$, ${ }^{* *} P<0.01$.

significantly suppressed the blood glucose elevation after gavaging glucose 30 minutes. At 60 minutes, significantly lower glucose levels were observed among almond oil groups and SSO group $(P<0.05)$. The glucose levels of LAO and MAO were significantly reduced at 120 minutes compared with the MC group $(P<0.05)$.

The AUC levels were presented in Figure 2(c). The MC group had a significantly higher level of glucose AUG compared with the CON group $(P<0.01)$. Significantly lower AUC levels were observed in LAO and MAO groups $(P<0.01, P<0.05)$ compared with the MC group.

Based on the results of blood glucose, changes in insulin levels in serum were also detected. As shown in Figure 2(d), the insulin level was significantly reduced in STZ-induced rats compared with the CON group $(P<0.01)$; however, the amount of insulin released was significantly increased in the LAO and MAO groups $(P<0.05, P<0.05)$. These results showed that the AO treatment could effectively maintain glucose homeostasis in STZ-induced rats.

\subsection{Effect of Almond Oil on the Oxidative and Inflammation-} Related Factors in Diabetic Rats. The results of oxidative stress in the serum are shown in Figure 3. The STZ-induced rats had significantly lower SOD and CAT antioxidant enzymes activity and higher MDA level $(P<0.01)$, while almond oil administration could reverse the changes. Treatments with almond oil for four weeks and the activity levels of SOD in LAO, SSO $(P<0.01, P<0.01)$, and MAO, HAO groups $(P<0.05, P<0.05)$ were increased compared with MC group; levels of CAT in LAO and MAO groups were increased $(P<0.05, P<0.05)$. MDA level in LAO and MAO groups $(P<0.01, P<0.01)$ and $\mathrm{HAO}$ and $\mathrm{SSO}$ groups $(P<0.05, P<0.05)$ were decreased compared with MC group. The results suggested that almond oil had the ability

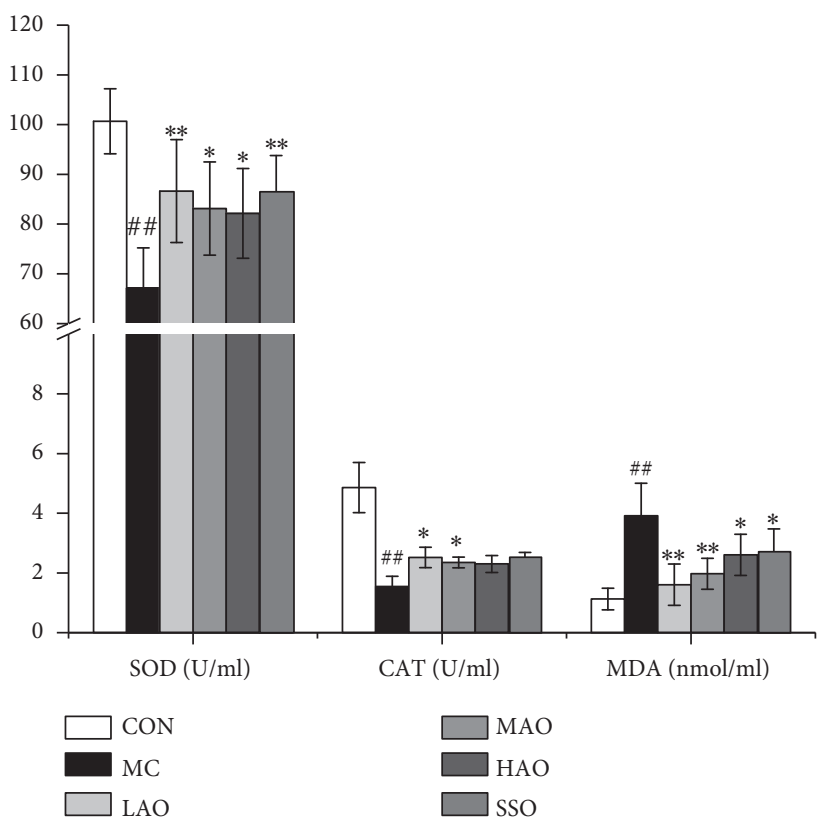

Figure 3: Effect of almond oil on serum oxidative status in STZinduced rats. SOD, superoxide dismutase; CAT, catalase; MDA, malondialdehyde. Data were expressed as means \pm SD. The MC group was compared with the CON group, ${ }^{\#} P<0.05$, ${ }^{\# \#} P<0.01$; the $\mathrm{LAO}, \mathrm{MAO}, \mathrm{HAO}$, and SSO groups were compared with the MC group, ${ }^{*} P<0.05,{ }^{* *} P<0.01$.

to suppress oxidative stress via increasing the activity of antioxidant enzymes and inhibiting lipid peroxidation in diabetic rats.

As shown in Figure 4, the levels of inflammatory factors were significantly elevated in the MC group compared with the CON group. TNF- $\alpha$ and IL- $1 \beta$ levels were significantly reduced in LAO, MAO, and HAO groups $(P<0.01$, 


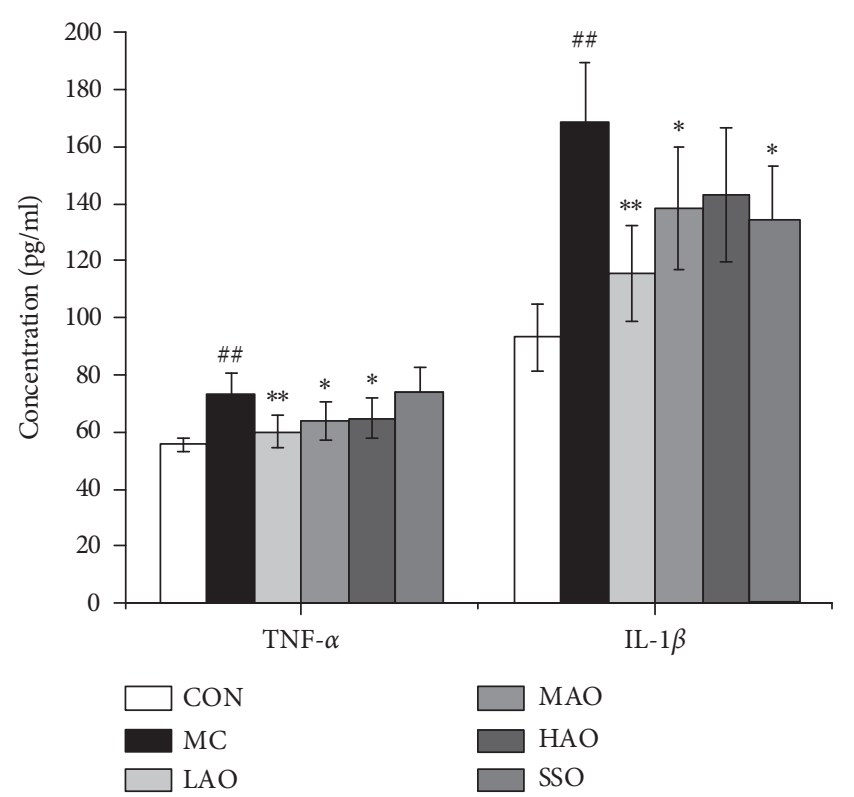

FIGURE 4: Effects of almond oil on serum TNF- $\alpha$ and IL- $1 \beta$ in STZinduced rats. TNF- $\alpha$, tumor necrosis factor- $\alpha$; IL- $1 \beta$, interleukin $1 \beta$. Data were expressed as means $\pm \mathrm{SD}$. The $\mathrm{MC}$ group was compared with the CON group, ${ }^{\#} P<0.05,{ }^{\# \#} P<0.01$; the LAO, $\mathrm{MAO}, \mathrm{HAO}$, and SSO groups were compared with the MC group, ${ }^{*} P<0.05,{ }^{* *} P<0.01$.

$P<0.05, P<0.05)$ and LAO, MAO, and SSO groups $(P<0.01, P<0.05, P<0.05)$ compared with MC group, respectively.

3.4. Effect of Almond Oil on Biochemical Parameters in Diabetic Rats. As shown in Figure 5, the levels of ALT, AST, CRE, BUN, and AGEs were significantly increased $(P<0.01)$ after STZ-induced diabetes in rats compared with the CON group. The levels of serum ALT and AST are important indicators of liver damage. The three doses of AO and SSO treatment groups significantly decreased ALT levels compared with the MC group $(P<0.01, P<0.05, P<0.05$, $P<0.05)$, and AST level was significantly reduced in LAO and MAO groups $(P<0.05, P<0.05)$. BUN, CRE, and AGEs are also indicators of kidney damage. As shown in Figure 6, the levels of BUN, CRE, and AGEs in STZ-induced diabetic rats were significantly upregulated compared with the CON group $(P<0.01)$. Conversely, lower levels of BUN and CRE were observed in LAO and MAO groups $(P<0.05, P<0.05)$ and LAO group $(P<0.05)$ compared to those of the MC group, respectively. AGEs level was significantly enhanced in all AO and SSO treatment groups $(P<0.01, P<0.05$, $P<0.05, P<0.05)$. The results demonstrated that almond oil treatment could alleviate liver and kidney dysfunctions.

\subsection{Effects of Almond Oil on Nrf2/HO-1 Pathway in Diabetic} Rats. To determine whether almond oil affects the antioxidant ability in diabetes through the $\mathrm{Nrf} 2 / \mathrm{HO}-1$ pathway, the expressions of Keap1, Nrf2, HO-1, and NQO1 were measured by Western blot. As shown in Figure 7, compared with

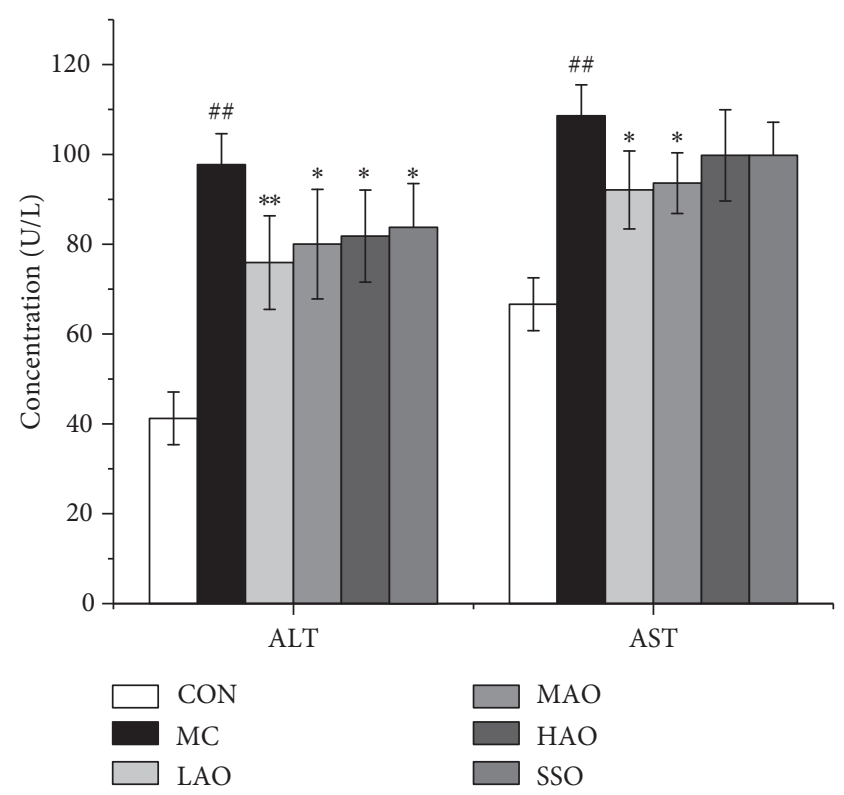

FIgURE 5: Effects of almond oil on serum ALT and AST in STZinduced rats. ALT, alanine transaminase; AST, aspartate transaminase. Data were expressed as means $\pm \mathrm{SD}$. The $\mathrm{MC}$ group compared with the CON group, ${ }^{\#} P<0.05$, ${ }^{\# \#} P<0.01$; the LAO, $\mathrm{MAO}, \mathrm{HAO}$, and SSO groups compared with the MC group, ${ }^{*} P<0.05,{ }^{* *} P<0.01$.

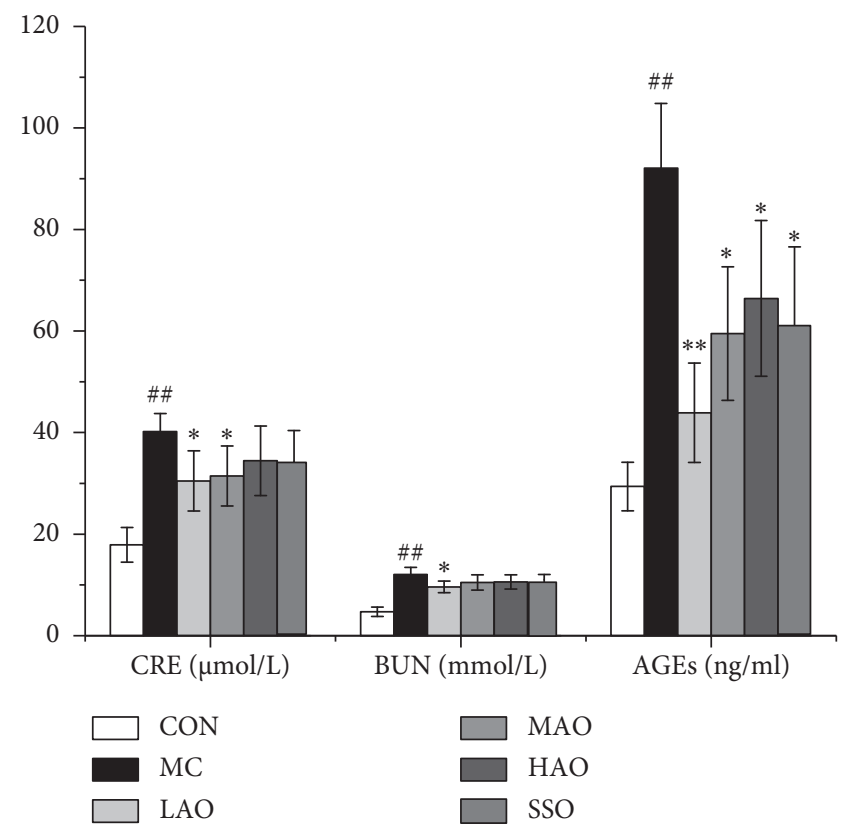

FIGURE 6: Effects of almond oil on serum CRE, BUN, and AGEs in STZ-induced rats. CRE, creatinine; BUN, blood urea nitrogen; AGEs, advanced glycation end products. Data were expressed as means $\pm \mathrm{SD}$. The MC group compared with the CON group, ${ }^{\#} P<0.05,{ }^{\#} P<0.01$; the LAO, MAO, HAO, and SSO groups compared with the MC group, ${ }^{*} P<0.05,{ }^{* *} P<0.01$.

the CON group, the expressions of Nrf2 and HO- $1(P<0.01$, $P<0.01)$ were significantly downregulated while the expression of Keap1 $(P<0.01)$ was upregulated in STZ-induced rats, which showed that the Nrf2 antioxidant signaling 

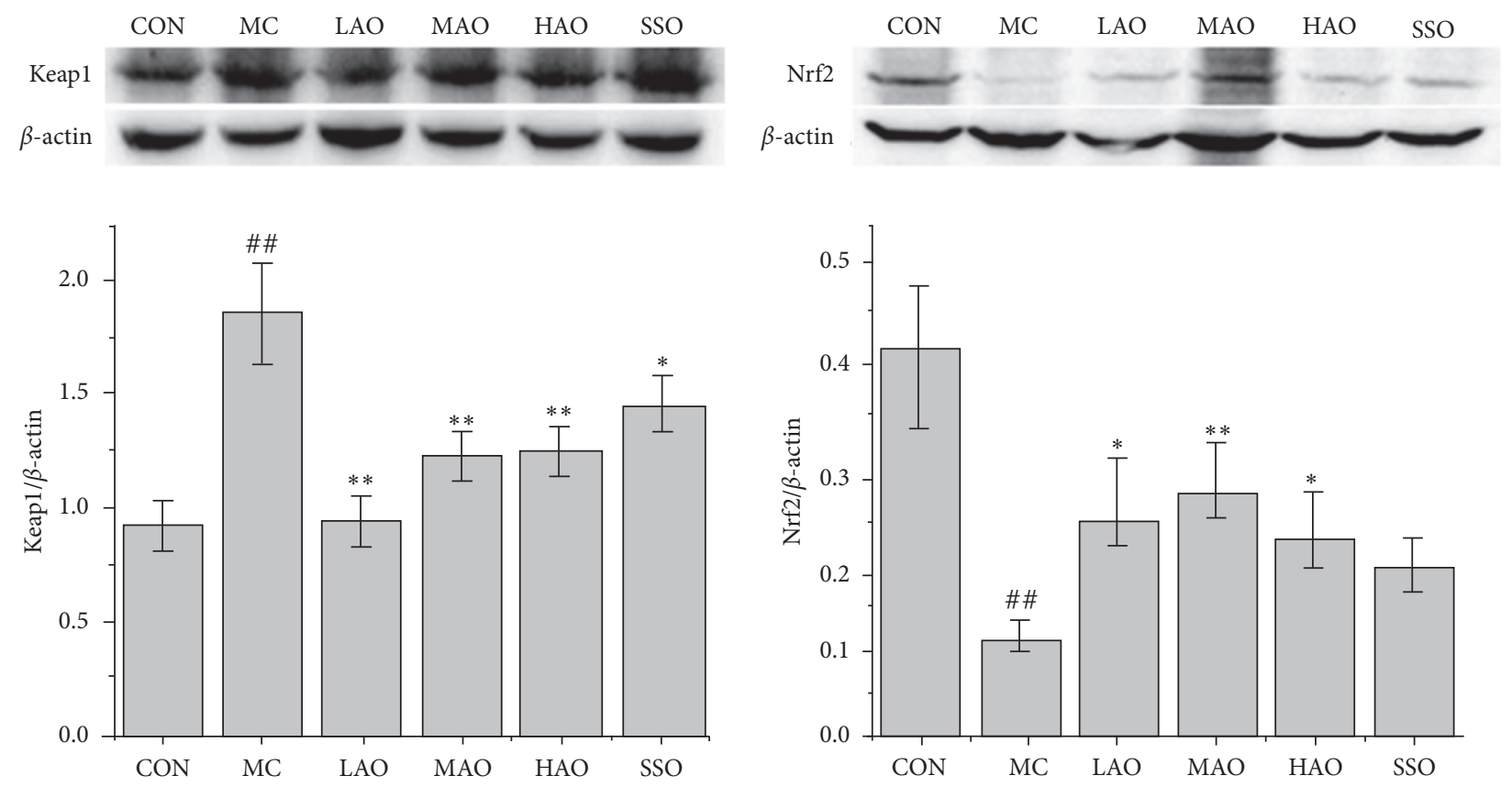

(a)
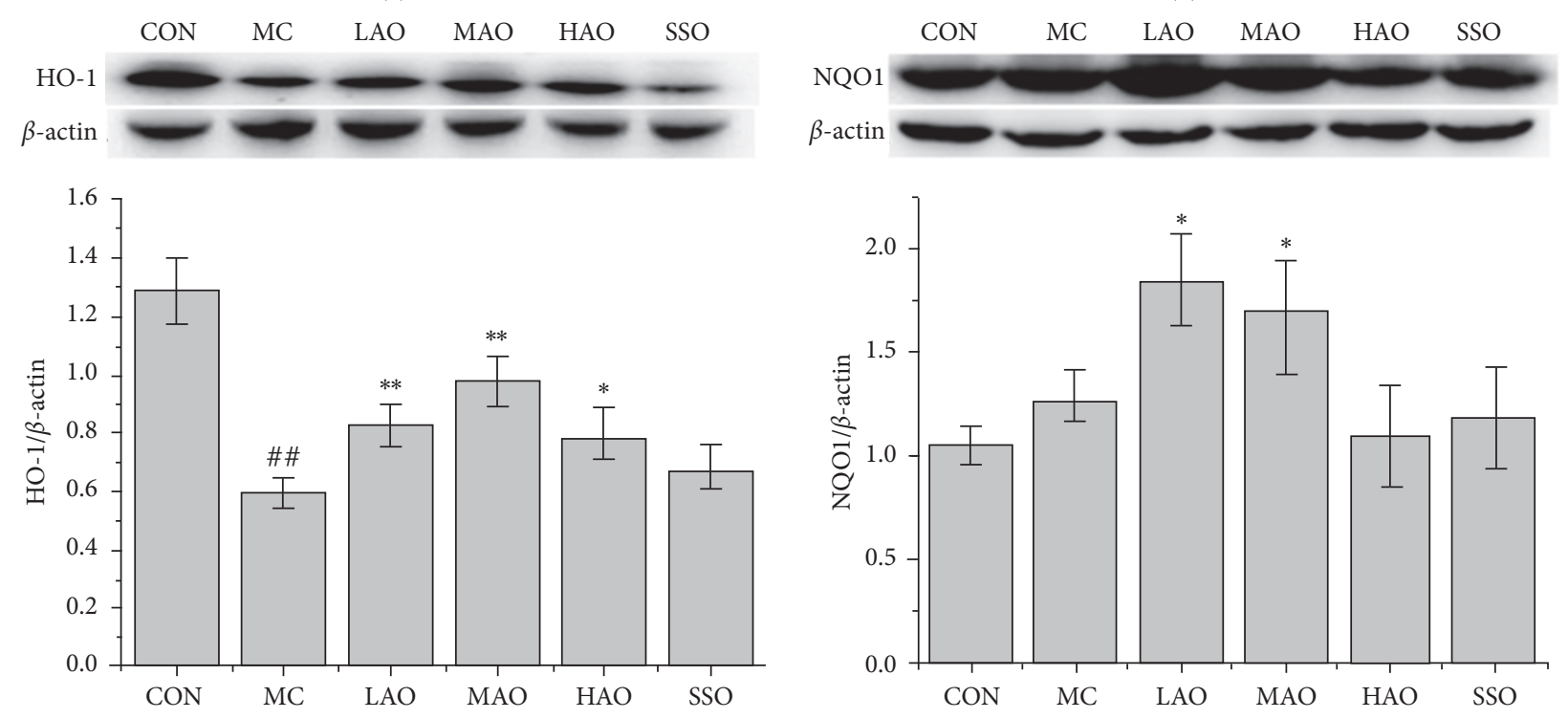

(c)

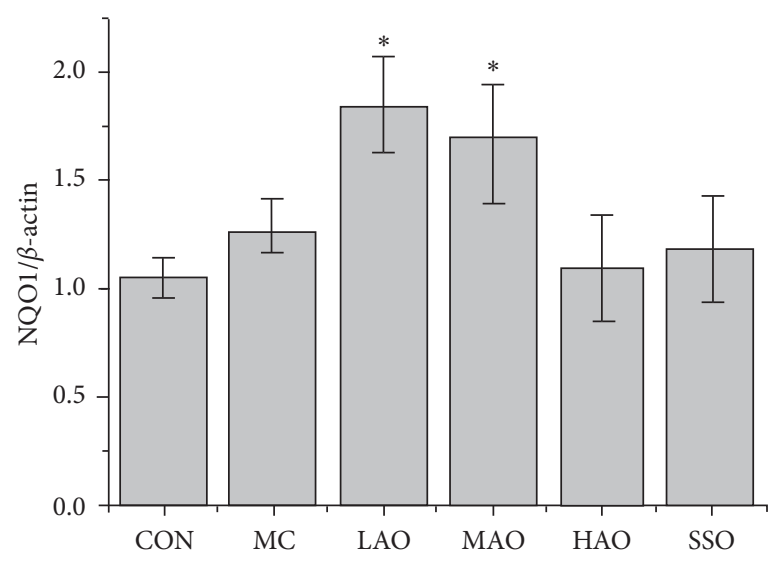

(d)

Figure 7: Effects of almond oil on Nrf2/HO-1 pathway in STZ-induced rats. Data are Western blotting results and gradation analysis of Keap1 (a), Nrf2 (b), HO-1 (c), and NQO1 (d). Data were expressed as means \pm SD. The MC group compared with the CON group, ${ }^{\#} P<0.05$, ${ }^{\# \#} P<0.01$; the LAO, MAO, HAO, and SSO groups compared with the MC group, ${ }^{*} P<0.05,{ }^{* *} P<0.01$.

pathway was impaired. Conversely, treatment with almond oil could reverse the changes compared with the MC group. Nrf2, HO-1, and NQO1 expressions were significantly upregulated in LAO, MAO, and HAO groups $(P<0.05$, $P<0.01, P<0.05)$, LAO, MAO, and HAO groups $(P<0.01$, $P<0.01, P<0.05)$, and LAO and MAO groups $(P<0.05$, $P<0.05)$. Keap1 expression was significantly downregulated in LAO, MAO, HAO, and SSO groups $(P<0.01, P<0.01$, $P<0.01, P<0.05)$. However, no significant changes in Nrf2, HO-1, or NQO1 expressions were observed between the SSO and $\mathrm{MC}$ groups. The results demonstrated that almond oil treatment could protect against oxidative-mediated injury by activating the $\mathrm{Nrf} 2 / \mathrm{HO}-1$ signaling pathway, which was correlated to its antioxidant effects.

\subsection{Almond Oil Modified the Gut Microbiota Structure.} The imbalance of interactions between host and gut microbiota could cause many important metabolic disorders, such as diabetes. In order to assess the effects of AO on the modulation of gut microbiota composition in diabetic rats, colon digesta samples were analyzed by $16 \mathrm{~S}$ rDNA 
sequencing. The effects of $\mathrm{AO}$ treatments on the changes of gut microbiota are shown in Figures 8-10.

The different microbial composition with overlaps was observed in the Venn diagram (Figure 8). Specifically, the CON group had 542 OTUs, MC group had 673 OTUs, SSO group had 584 OTUs, and the AO groups (LAO, MAO, and HAO) had 689, 648, and 518 OTUs, respectively. 331 OTUs were shared by these groups.

As shown in 1, compared with the CON group, Chao 1, Ace, and Shannon indices $(P<0.01, P<0.01)$ were significantly increased, while Simpson index $(P<0.01)$ was decreased in STZ-induced rats.

In the beta diversity analysis, the principal coordinate analysis (PCoA) was used to reflect the compositional differences among groups. As shown in Figure 10, there was a clear separation of gut microbiota composition between the CON and $\mathrm{MC}$ groups, and the microbial composition in the almond oil treatment groups was more close to that in the CON group.

We analyzed the phylum and genus level characteristics further to explore the gut microbiota structure differences among groups. The relative abundance in the phylum level was shown in Figure 10(a). In the CON group, the dominant phyla were Firmicutes (60.74\%) and Bacteroidetes (26.79\%). Compared with the CON group, the abundance of Firmicutes increased to $72.57 \%$, while the Bacteroidetes significantly decreased to $16.85 \%$ in the MC group $(P<0.05)$. However, the consumption of almond oil modified the gut microbiota. Specifically, the middle dose of almond oil decreased the abundance of Firmicutes to $62.87 \%$ and significantly increased Bacteroidetes to $21.89 \%(P<0.05)$.

At the genus level, the microbiota of the MC group changed greatly compared to that of the normal rats (Figure 10(b)). The abundances of Ruminococcaceae_UCG014, Clostridium_sensu_stricto_1, and Fusicatenibacter were increased, while Lactobacillus, Bacteroides, and Lachnospiraceae_NK4A136_group were decreased in the MC group compared with the CON group. Different doses of AO treatment can partially restore the microbiota change. The high dose of AO decreased Ruminococcaceae_UCG-014 $(P<0.05)$, the low dose of AO decreased Clostridium_sensu_stricto_1 and Fusicatenibacter $(P<0.05$, $P<0.05)$, the high dose of AO increased Lactobacillus and Lachnospiraceae_NK4A136_group $(P<0.05, P<0.05)$, and the middle dose of AO increased Bacteroides $(P<0.05)$.

3.7. Spearman's Correlation Analysis. Spearman's correlation analysis was used to further explore the relationship between gut microbiota and metabolic parameters (Figure 11). We analyzed the correlation between bacteria genus, FBG, insulin (INS), body weight (BW), SOD, CAT, MDA, TNF- $\alpha$, IL- $1 \beta$, Keap1, Nrf2, HO-1, and NQO1. The abundances of Clostridium_sensu_stricto_1 and Fusicatenibacter were positively correlated with FBG, MDA, TNF- $\alpha$, IL- $1 \beta$, and Keap1, while being negatively correlated with INS, BW, SOD, CAT, Nrf2, and HO-1. The abundance of Ruminococcaceae_UCG-014 was negatively correlated with BW, SOD, Nrf2, and HO-1. The abundance of Lachnospiraceae_NK4A136_group was positively correlated with SOD and Nrf2, while being

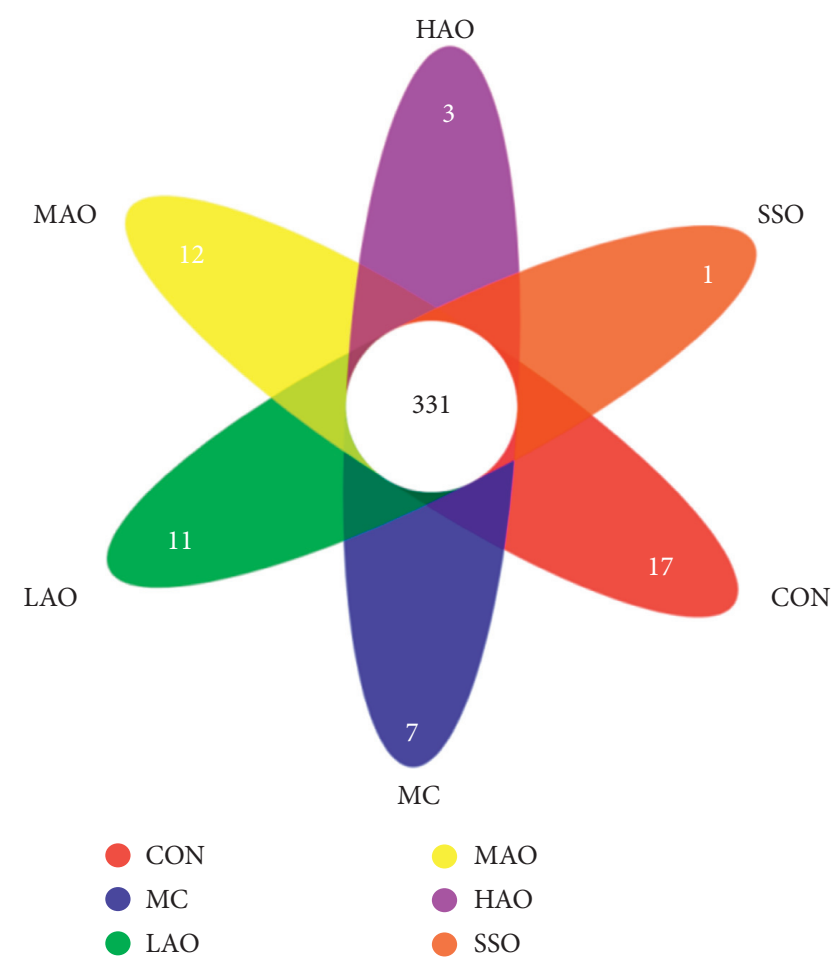

FIgURE 8: Venn diagrams of the OTUs between different groups.

negatively correlated with FBG. The abundance of Bacteroides was positively correlated with INS, SOD, CAT, Nrf2, and HO1 , while being negatively correlated with FBG and IL-1 $\beta$. These bacteria genus changes were relevant to the regulations of glucose metabolism, oxidative stress, and inflammation. The result showed that almond oil treatment could recover some relevant dysbiosis of the gut microbiota and regulate the glucose metabolism and Nrf2/HO-1 pathway.

\section{Discussion}

As a chronic metabolic disease, diabetes has proven to be a major threat to health risks. In recent years, the diet has been considered a crucial tool in the prevention and protection from disease and its complications, and dietary lipids have been regarded as one of the most necessary macronutrients for human health. Searching for dietary lipids with antidiabetic activity and few side effects has been essential to control the development of diabetes. In the present study, we exhibited that oral administration of almond oil for 4 weeks alleviated the symptoms of diabetes induced by STZ.

STZ is commonly used to induce experimental diabetes in rodents. STZ enters pancreatic $\beta$-cells through the GIUT2 glucose transporter [25] and induces the production of ROS, which rapidly and irreversibly destroys pancreatic $\beta$ cells $[26,27]$. As the characteristics of diabetes, polyphagia, polydipsia, hyperglycemia, and reduced BW were observed after STZ induction, which were demonstrated in previous research [28].

The main bioactive compound of almond oil is the oleic $(n-9)$ fatty acids. Previous studies found that $n-9$ fatty acids could reduce the local inflammatory response [29]. Oleic 


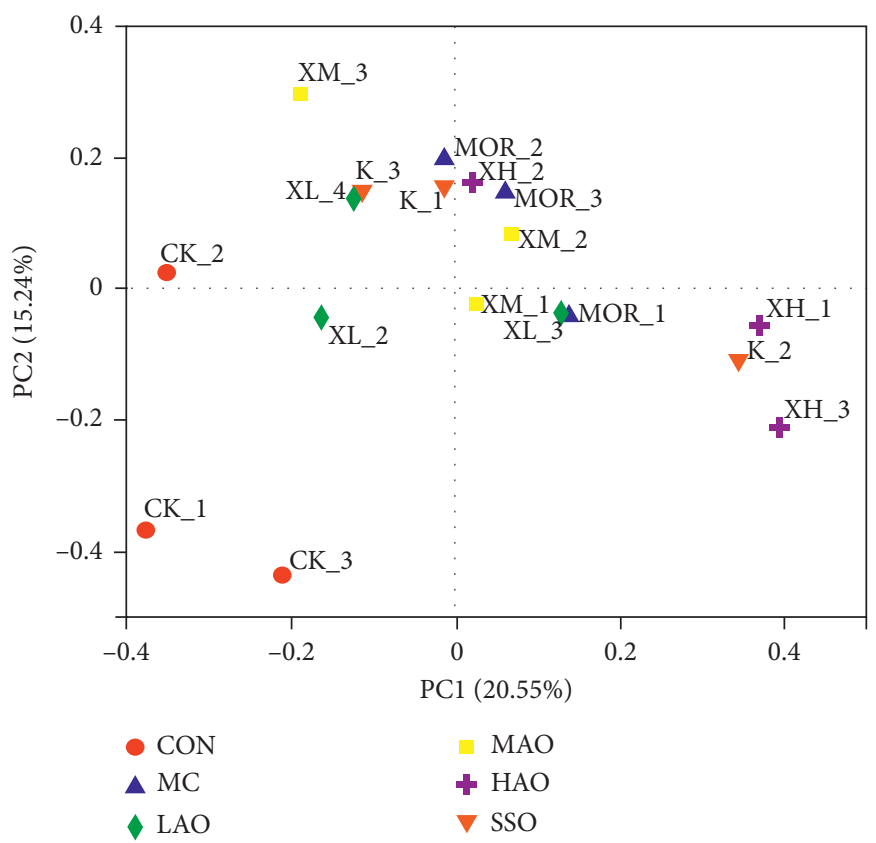

FiguRE 9: Principal coordinate analysis (PCoA) between different groups.
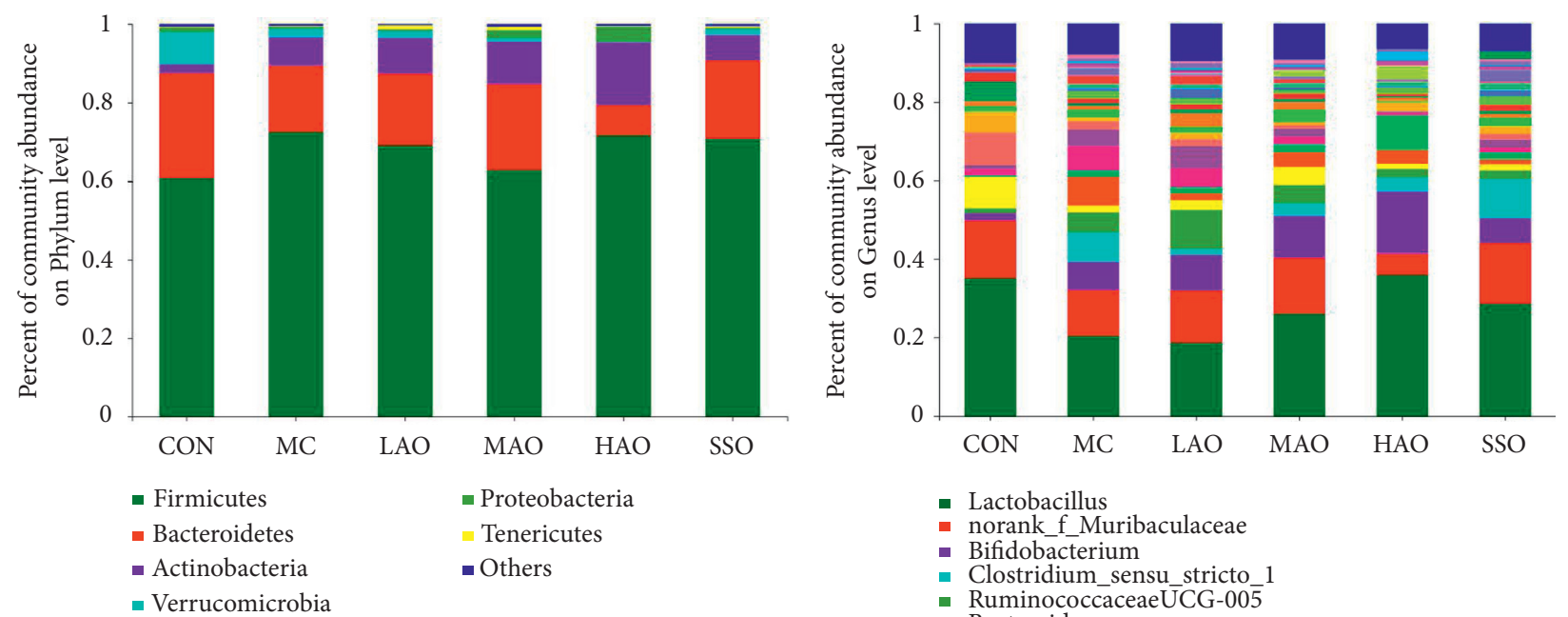

- Lactobacillus

- norank_f_Muribaculaceae

- Bifidobacterium

- Clostridium_sensu_stricto_1

- RuminococcaceaeUCG-005

- Bacteroides

- Fusicatenibacter

- Ruminococcaceae_UCG-014

- Ruminococcus 1

- Akkermansia

- Lachnospiraceae_NK4A136_group

- norank_f_Ruminococcaceae

- unclassified $\mathrm{f}$ Lachnospiraceae

- unclassified f Ruminococcaceae

- Alistipes

- Turicibacter

- (Eubacterium)_coprostanoligenes_group

- Christensenellaceae_R-7_group

- Ruminiciostridum_6

- CAG-352

- Escherichia-Shigella

- unclassified_p_Firmicutes

- Faecalibaculum

- Ruminococcaceae UCG-013

- Prevotellaceae UCG-001

- Coprococcus_2

- Others the phylum level. (b) The effect of almond oil on relative abundances at the genus level. 
TABle 1: Effects of almond oil on Alpha diversity.

\begin{tabular}{lcrrr}
\hline \multirow{2}{*}{ Group } & \multicolumn{2}{c}{ Community richness } & \multicolumn{2}{c}{ Community diversity } \\
& Chao 1 & Ace & Simpson & Shannon \\
\hline CON & $416.97 \pm 44.77$ & $419.59 \pm 46.40$ & $0.3175 \pm 0.1282$ & $2.6319 \pm 0.3733$ \\
MC & $541.84 \pm 21.36^{\# \#}$ & $533.70 \pm 24.61^{\#}$ & $0.0713 \pm 0.0253^{\#}$ & $3.7212 \pm 0.2476^{\#}$ \\
LAO & $475.53 \pm 69.38$ & $480.15 \pm 77.50$ & $0.0802 \pm 0.0335$ & $3.6362 \pm 0.4064$ \\
MAO & $428.57 \pm 51.25^{* *}$ & $424.33 \pm 45.06^{* *}$ & $0.1277 \pm 0.0902$ & $3.3834 \pm 0.5983$ \\
HAO & $395.07 \pm 33.71^{* *}$ & $394.07 \pm 39.80^{* *}$ & $0.1804 \pm 0.1007$ & $2.8502 \pm 0.6498$ \\
SSO & $480.08 \pm 58.78$ & $481.47 \pm 55.62$ & $0.1325 \pm 0.1546$ & $3.5032 \pm 0.9386$ \\
\hline
\end{tabular}

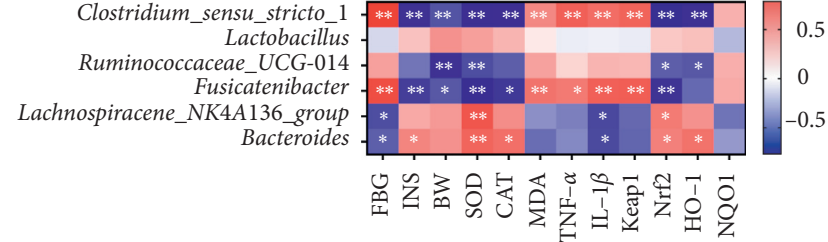

FIGURE 11: Significant correlation between the abundance of bacteria genus and metabolic parameters was selected using Spearman correlation analysis $\left({ }^{*} P<0.05,{ }^{* *} P<0.01\right)$.

acid also has a cytoprotective effect for $\beta$-cells, and dietary MUFAs have the potential to act as regulators of glucose homeostasis in either the inner-digestive or postabsorptive period [30]. Previous research demonstrated that oleic acid could suppress the deleterious effects of palmitic acid on the insulin signaling pathway [31]. In this study, we chose sunflower seed oil as the positive group. Sunflower seed oil is one of the most consumed dietary sources of polyunsaturated fatty acids and a kind of dietary lipids, which is widely used in the Chinese diet.

Chronic hyperglycemia leads to the excessive generation of ROS, such as hydrogen peroxide $\left(\mathrm{H}_{2} \mathrm{O}_{2}\right)$, superoxide $\left(\mathrm{O}_{2}^{-}\right)$, hydroxyl $(\mathrm{OH})$, or peroxyl $(\cdot \mathrm{OOH})$ radicals through glucose autoxidation [32]. Oxidative stress occurs as the excessive accumulation of ROS exceeding local antioxidant capacity, which may damage cellular organelles and causes an increase in lipid peroxidation [33]. More importantly, the oxidative status level, which plays a crucial role in the antioxidant system, was evaluated by measuring MDA, SOD, and CAT activities in our study. SOD is an essential endogenous antioxidant enzyme used to eliminate superoxide radicals and protect cells against the toxic byproducts of aerobics metabolism [34]. SOD could convert superoxide radicals to hydrogen peroxide $\left(\mathrm{H}_{2} \mathrm{O}_{2}\right)$, which is decomposed into oxygen and water by catalase, and maintain the oxidation balance. Polyunsaturated fatty acids in the cell membrane were attacked by free radicals and formed MDA. MDA was formed by the attacking free radicals of polyunsaturated fatty acids in the cell membrane. As an oxidation end product and a biomarker of lipid peroxidation, MDA could indirectly reflect the extent of oxidative damage in the body [35]. The present study found that SOD and CAT activity were significantly decreased, and MDA content was increased in the MC group rats treated by STZ. In contrast, the results were reversed after almond oil treatment. Consistent results have proved that almond oil had an antioxidant modulating effect on rats with hepatic injury [24].
Oxidative stress has a crucial effect on the inflammatory reaction, which increases the excretion of inflammation-related cytokines [36]. TNF- $\alpha$ and IL- $\beta$ are considered the central proinflammatory mediators for the inflammatory reaction, which are the major parts of the inflammatory process leading to $\beta$-cell destruction and closely associated with the progression of diabetes [37]. TNF- $\alpha$ is a cytotoxin mainly produced by monocytes and macrophages and could induce the activation and accumulation of IL-1 $\beta$, stimulating the immune disorder [38]. IL-1 $\beta$ affects the activation of nuclear factor kappa B (NF- $\kappa \mathrm{B})$, promoting the expression of several $\beta$-cells genes [39]. The overproduction of IL- $1 \beta$ leads to the pathogenesis of inflammation and autoimmune diseases and correlates with the recruitment of immune cells and $\beta$-cell damage in islets [40]. In our study, almond oil treatment significantly reduced the levels of TNF- $\alpha$ and IL- $1 \beta$ induced by STZ, suggesting that it had the potential effects of suppressing the expression of proinflammatory cytokines. However, no significant change of TNF- $\alpha$ was observed between the sunflower seed oil treatment group and the STZ-induced group. The sunflower seed oil contains about $67 \%$ linoleic acid (LA). LA is a direct precursor of the proinflammatory arachidonic acid (AA). AA can participate in cell signaling and trigger inflammation, which is linked to oxygen-free radical rise and results in more severe reactions in some tissues [41]. In contrast, the inflammatory markers of people with high MUFAs dietary habits have been considerably reduced, and oleic acid can reverse the inhibitory effect of TNF- $\alpha$ on insulin production, revealing that oleic acid has the potential therapeutic effect in an inflammatory context [42]. In summary, the anti-inflammatory effect of almond oil was better than sunflower seed oil according to the results of inflammatory factors due to its high content of oleic acid probably.

The chronic accumulation of circulating glucose might cause damage to the whole body blood vessels, which affected the functionality of some vital organs and increased the risk of various life-threatening health complications, such as chronic liver and kidney disease. ALT and AST activities may reflect the damage of hepatocytes and the markers of hepatocyte integrity. The contents of ALT and AST are low in the serum of normal rats but released into the blood due to the damage of hepatocytes or cell membrane permeability. In the present study, ALT and AST levels were increased in diabetes groups induced by STZ. Simultaneously, the administration of almond oil significantly reduced those levels, which was consistent with the result that treatment with almond oil suppressed the acute hepatic damage [23]. 
Hyperglycemia is characterized by the high content of glycated proteins and binding of monosaccharides to amino groups of proteins, leading to the formation of AGEs and changes in the structure and functions of proteins. Besides, glycated proteins can activate membrane receptors by glycation end products and induce intracellular oxidative stress and a proinflammatory status [43], consequently affecting kidney functions. CRE and BUN are also the biochemical markers of kidney functions. In the present study, the increased CRE, BUN, and AGEs levels in diabetic rats were reversed by almond oil treatment, which were consistent with the result that administration with almond oil changed the levels of CRE and BUN in rats exposed to a sublethal concentration of lead [44].

$\mathrm{Nrf} 2 / \mathrm{HO}-1$ is the main signaling pathway for antioxidative stress [12]. Under normal conditions, Nrf2 is bound by Keap 1 in the cytoplasm and then degraded by the ubiquitination system. Under oxidative stress or inflammatory conditions, Nrf2 is released from Keap1 and translocated into the nucleus, where it combines with antioxidant response elements [10], upregulates the transcription of HO-1 and NQO1, and then participates in the antioxidation processes. HO- 1 is a redox-sensitive inducible stress protein activated by Nrf2. HO-1 degrades heme into biliverdin, free iron, and carbon monoxide, which exerts a protective role by reducing oxidative injury and attenuating inflammatory response [45]. Our results found that the antioxidant mechanism of almond oil appeared to activate Nrf2 signaling pathway and upregulate the downstream of Nrf2. The consistent result also found that taurine could reduce the severity of oxidative stress by activating the antioxidative defense signaling pathway in diabetic rats [46]. The results also showed that the antioxidant ability of almond oil was better than sunflower seed oil.

Previous studies suggested that the blood glucose levels were also regulated in the intestine, which was a major organ involved in the glucose homeostasis, carbohydrate digestion, and secretion of glucose into the circulation, and the energy substrates can be used by intestinal colonized microbiota [4]. It indicated that gut microbiota has a protective effect on metabolism regulation and has a positive effect on glucose metabolism [47].

The Chao 1 and Ace indices were used to reflect the richness of the communities; the Simpson and Shannon indices were used to reflect the diversity of the communities. The richness and diversity of gut microbiota in STZ-induced rats were increased compared with the CON group, which were consistent with the previous report. The increase of gut microbiota diversity and richness could be associated with the development of disease [48].

Firmicutes and Bacteroidetes were the most abundant phyla in normal rats. The ratio of Firmicutes/Bacteroidetes was widely regarded as an indicator of gut microbiota dysbiosis in many metabolic diseases [49]. A decrease in Bacteroidetes has been associated with pathologies such as inflammatory bowel disease and diabetes [50]. A reduced relative abundance of Bacteroidetes and an increase in Firmicutes were observed in STZ-induced rats, resulting in an increased ratio of Firmicutes/Bacteroidetes, which were consistent with the previous study [4]. However, the ratio was downregulated upon treatment with almond oil.

Lactobacillus can promote glucose metabolism through directly consuming glucose in the host intestines [51]. Lactobacillus can inhibit the growth of acid-sensitive pathogenic bacteria by lowering the $\mathrm{pH}$ of the intestinal environment. It also competes with pathogens to protect the gut barrier and immune response of the host and improves intestinal oxidative stress in rats with liver damage [52]. In our study, the abundance of Lactobacillus was decreased in the MC group, while the change was reversed by oral administration of almond oil, which was in line with the previous report that dietary administration with pistachio nuts increased the abundance of Lactobacillus in diabetic rats [53]. Some bacteria in Ruminococcaceae are proinflammatory bacteria and associated with inflammatory bowel diseases [54]. The previous research analyzed the correlation between gut microbiota and blood glucose, and results showed that Ruminococcaceae_UCG-014 had a positive correlation with the occurrence and development of diabetes [55]. In this study, the abundance of Ruminococcaceae_UCG-014 was increased in the MC group, while the level of Ruminococcaceae_UCG-014 was decreased by almond oil. Clostridium_sensu_stricto_1 is considered as an LPS-producing pathogenic bacteria associated with intestinal inflammation and infection [56, 57]. High level of LPS accumulates in the intestine and participates in the inflammation of diabetics. Reducing the level of LPS-producing bacteria could reduce harmful substances from the intestines into the blood and inhibit the incidence of oxidative stress and inflammation [58]. Clostridium_sensu_stricto_1 and Fusicatenibacter were positively correlated with FBG levels. Our study also found that the abundance of Clostridium_sensu_stricto_1 and Fusicatenibacter was decreased by almond oil treatment compared to the MC group, which was in accordance with the report that propolis decreased the abundance of Clostridium_sensu_stricto_1 and Fusicatenibacter in diabetic rats [59]. Lachnospiraceae_NK4A136_group and Bacteroides are both shortchain fatty acids- (SCFA-) producing intestinal bacteria, which enhance the gut barrier function [60]. Lachnospiraceae_NK4A136_group inhabits the human gut and produces butyric acid that is considered as an intestinal barrier protector, which is associated with maintaining intestinal health [61]. Bacteroides is beneficial bacteria that plays an important role in glucose metabolism in the host [62]. The present study found that almond oil increased the abundances of Lachnospiraceae_NK4A136_group and Bacteroides compared with the MC group induced by STZ, which were consistent with the report that Lycium barbarum alleviated gut microbiota dysbiosis [63]. The results indicated that almond oil could reduce the relative abundance of proinflammatory bacteria and enhance the butyrate-producing bacteria, which alleviated abnormal glucose metabolism. Our results showed that the intake of low dose almond oil presented the best protective effect; however, the high dose almond oil presented a better impact on the antidysbiosis of gut microbiota. According to the correlation analysis, the abundance of Clostridium_sensu_stricto_1 and 
Fusicatenibacter was positively correlated with oxidative stress and inflammatory response, while being negatively correlated with glucose metabolism and antioxidant enzymes activity. The abundance of these two genera was significantly reduced in the LAO group. The LAO group might regulate the main pathogenic bacteria to reduce the levels of oxidative stress and proinflammatory factors.

Our study suggested that almond oil administration for four weeks could positively regulate the gut microbiota of the host and inhibit the blood glucose elevation through increasing beneficial bacteria and reducing colonization of potentially pathogenic bacteria in metabolism pathways. Whether almond oil could increase the levels of SCFAs and maintain intestinal barrier function should be studied in further research.

\section{Conclusions}

In conclusion, almond oil alleviated the development of STZ-induced diabetes in rats.

The administration of almond oil significantly decreased the levels of FBG, promoted insulin secretion, increased BW and the ability to glucose tolerance, suppressed oxidative stress and inflammatory reaction, and improved liver and kidney function. The beneficial effect of almond oil appeared to be associated with activating $\mathrm{Nrf} / \mathrm{HO}-1$ pathway against oxidative stress and regulating gut microbiota on glucose metabolism.

These results showed that the effect of almond oil on ameliorating diabetes is associated with its antioxidative and anti-inflammatory properties in STZ-induced diabetic rats, which indicated that replacing the common oil in the diet with almond oil may have a potential beneficial effect on diabetes, suggesting that almond oil could be used as an adjunct treatment strategy for diabetes.

\section{Data Availability}

The data used to support the findings of this study are available from the corresponding author upon request.

\section{Ethical Approval}

All the experimental procedures and animal welfare were in accordance with the related ethical regulation guidelines and approved by the Research Ethics Committee of the College of Food Science and Technology in Hebei Agricultural University (2017-006).

\section{Conflicts of Interest}

The authors declare that they have no conflicts of interest.

\section{Acknowledgments}

This work was supported by the Hebei Province Modern Agricultural Industrial Technology System Sheep Industry Innovation Team Special (No. HBCT2018140203); the Food Processing Discipline Group of Hebei Agricultural University (No. 2020-04); and the Research and Demonstration of the Key Technology of Using Nanocellulose Stabilized Pickering Emulsion Substitute Fat In Low-Fat Meat Products (No. 20327116D).

\section{Supplementary Materials}

The fatty acid composition analysis of almond oil was shown in Table S1 in the Supplementary Material. (Supplementary Materials)

\section{References}

[1] Y. Xu, L. M. Wang, and J. He, "Prevalence and control of diabetes in Chinese adults," Jama, vol. 310, no. 9, pp. 948-958, 2013.

[2] N. H. Cho, J. E. Shaw, S. Karuranga et al., "IDF Diabetes Atlas: global estimates of diabetes prevalence for 2017 and projections for 2045," Diabetes Research and Clinical Practice, vol. 138, pp. 271-281, 2018.

[3] J. Xie, W. Song, X. C. Liang et al., "Protective effect of quercetin on streptozotocin-induced diabetic peripheral neuropathy rats through modulating gut microbiota and reactive oxygen species level," Biomedicine \& Pharmacotherapy, vol. 127, Article ID 110147, 2020.

[4] Y. Feng, H. Weng, L. Ling et al., "Modulating the gut microbiota and inflammation is involved in the effect of Bupleurum polysaccharides against diabetic nephropathy in mice," International Journal of Biological Macromolecules, vol. 132, pp. 1001-1011, 2019.

[5] M. Yang, Y. Chen, T. Zhao, and Z. J. Wang, "Effect of astaxanthin on metabolic cataract in rats with type 1 diabetes mellitus," Experimental and Molecular Pathology, vol. 113, Article ID 104372, 2020.

[6] A. Avogaro and G. P. Fadini, "The effects of dipeptidyl peptidase-4 inhibition on microvascular diabetes complications," Diabetes Care, vol. 37, no. 10, pp. 2884-2894, 2014.

[7] A. I. Vinik and E. J. Vinik, "Prevention of the complications of diabetes," The American Journal of Managed Care, vol. 9, no. 3, pp. 63-80, 2014.

[8] T. I. Adelusi, L. Du, M. Hao et al., "Keap1/Nrf2/ARE signaling unfolds therapeutic targets for redox imbalanced-mediated diseases and diabetic nephropathy," Biomedicine \& Pharmacotherapy, vol. 123, Article ID 109732, 2020.

[9] H. Murata, H. Takamatsu, and S. Lie, "NRF2 regulates PINK1 expression under oxidative stress conditions," PLoS One, vol. 10, no. 11, Article ID 0142438, 2015.

[10] S. Y. Wang, P. Nie, X. D. Lu et al., "Nrf2 participates in the anti-apoptotic role of zinc in type 2 diabetic nephropathy through Wnt/beta-catenin signaling pathway," The Journal of Nutritional Biochemistry, vol. 84, Article ID 108451, 2020.

[11] X. Cheng, R. C. M. Siow, and G. E. Mann, "Impaired redox signaling and antioxidant gene expression in endothelial cells in diabetes: a role for mitochondria and the nuclear factor-E2related factor 2-kelch-like ECH-associated protein 1 defense pathway," Antioxidants \& Redox Signaling, vol. 14, no. 3, pp. 469-487, 2011.

[12] J. Ren, D. Su, L. X. Li et al., "Anti-inflammatory effects of Aureusidin in LPS-stimulated RAW264.7 macrophages via suppressing NF-kappaB and activating ROS- and MAPKsdependent Nrf2/HO-1 signaling pathways," Toxicology \& Applied Pharmacology, vol. 387, Article ID 114846, 2020.

[13] A. L. Wang, D. C. Cai, H. Zhang et al., "Using herbal medicine to target the "microbiota-metabolism-immunity" axis as 
possible therapy for cardiovascular disease," Pharmacological Research, vol. 142, pp. 205-222, 2019.

[14] M. Knip and H. Siljander, "The role of the intestinal microbiota in type 1 diabetes mellitus," Nature Reviews Endocrinology, vol. 12, no. 3, pp. 154-167, 2016.

[15] Q. Zhang, Y. Pan, B. Zeng et al., "Intestinal lysozyme liberates Nod1 ligands from microbes to direct insulin trafficking in pancreatic beta cells," Cell Research, vol. 29, no. 7, pp. 516-532, 2019.

[16] R. Wirth, N. Bdi, G. Marti et al., "Regionally distinct alterations in the composition of the gut microbiota in rats with streptozotocin-induced diabetes," PLoS One, vol. 9, no. 12, Article ID 0110440, 2014.

[17] Y. Liu, C. Wang, J. Li et al., "Phellinus linteus polysaccharide extract improves insulin resistance by regulating gut microbiota composition," The FASEB Journal, vol. 34, no. 1, pp. 1065-1078, 2020.

[18] K. P. Scott, S. W. Gratz, P. O. Sheridan, H. J. Flint, and S. H. Duncan, "The influence of diet on the gut microbiota," Pharmacological Research, vol. 69, no. 1, pp. 52-60, 2013.

[19] S. S. K. Wijeratne, M. M. Abou-Zaid, and F. Shahidi, "Antioxidant polyphenols in almond and its coproducts," Journal of Agricultural and Food Chemistry, vol. 54, no. 2, pp. 312-318, 2006.

[20] C.-Y. Chen, K. Lapsley, and J. Blumberg, "A nutrition and health perspective on almonds," Journal of the Science of Food and Agriculture, vol. 86, no. 14, pp. 2245-2250, 2006.

[21] X. Chen, L. Li, X. Liu et al., "Oleic acid protects saturated fatty acid mediated lipotoxicity in hepatocytes and rat of non-alcoholic steatohepatitis," Life Sciences, vol. 203, pp. 291-304, 2018.

[22] Z. Ahmad, "The uses and properties of almond oil," Complementary Therapies in Clinical Practice, vol. 16, no. 1, pp. 10-12, 2010.

[23] X.-Y. Jia, Q.-A. Zhang, Z.-Q. Zhang et al., "Hepatoprotective effects of almond oil against carbon tetrachloride induced liver injury in rats," Food Chemistry, vol. 125, no. 2, pp. 673-678, 2011.

[24] L. R. D. O. Torres, F. C. D. Santana, F. L. Torres-Leal et al., "Pequi (Caryocar brasiliense Camb.) almond oil attenuates carbon tetrachloride-induced acute hepatic injury in rats: antioxidant and anti-inflammatory effects," Food and Chemical Toxicology, vol. 97, pp. 205-216, 2016.

[25] H. E. Eitah, Y. A. Maklad, N. F. Abdelkader, A. A. Gamal el Din, M. A. Badawi, and S. A. Kenawy, "Modulating impacts of quercetin/sitagliptin combination on streptozotocin-induced diabetes mellitus in rats," Toxicology and Applied Pharmacology, vol. 365, pp. 30-40, 2019.

[26] M. H. Jin, G. N. Shen, Y. H. Jin et al., "Peroxiredoxin I deficiency increases pancreatic $\beta$-cell apoptosis after streptozotocin stimulation via the AKT/GSK3 $\beta$ signaling pathway," Molecular Medicine Reports, vol. 22, no. 3, pp. 1831-1838, 2020.

[27] T. Szkudelski, "The mechanism of alloxan and streptozotocin action in Bcells of the rat pancrea," Physiological Research, vol. 50, no. 6, pp. 537-546, 2001.

[28] Y. Cheng, L. Sibusiso, L. Hou et al., "Sargassum fusiforme fucoidan modifies the gut microbiota during alleviation of streptozotocin-induced hyperglycemia in mice," International Journal of Biological Macromolecules, vol. 131, pp. 1162-1170, 2019.

[29] C. R. B. Cardoso, M. A. Souza, E. A. V. Ferro et al., "Influence of topical administration of n-3 and n-6 essential and n-9 nonessentia," Wound Repair and Regeneration, vol. 12, no. 2, pp. 235-243, 2004.

[30] S. López, B. Bermúdez, R. Abia, F. J. Muriana et al., "The influence of major dietary fatty acids on insulin secretion and action," Current Opinion in Lipidology, vol. 21, no. 1, pp. 15-20, 2010.

[31] V. Pardo, Á. González-Rodríguez, S. C. Kozma, and Á. M. Valverde, "Role of hepatocyte S6K1 in palmitic acidinduced endoplasmic reticulum stress, lipotoxicity, insulin resistance and in oleic acid-induced protection," Food and Chemical Toxicology, vol. 80, pp. 298-309, 2015.

[32] S. A. Sheweita, S. A. ElHady, and H. M. Hammoda, "Trigonella stellata reduced the deleterious effects of diabetes mellitus through alleviation of oxidative stress, antioxidantand drug-metabolizing enzymes activities," Journal of Ethnopharmacology, vol. 256, Article ID 112821, 2020.

[33] A. G. Jagtap and P. B. Patil, "Antihyperglycemic activity and inhibition of advanced glycation end product formation by Cuminum cyminum in streptozotocin induced diabetic rats," Food \& Chemical Toxicology, vol. 48, no. 8-9, pp. 2030-2036, 2010.

[34] B. Mansuroğlu, S. Derman, A. Yaba, and K. Kızılbey, "Protective effect of chemically modified SOD on lipid peroxidation and antioxidant status in diabetic rats," International Journal of Biological Macromolecules, vol. 72, pp. 79-87, 2015.

[35] S. Nabi, "Promotion of peroxidation and hydroperoxidation of lipids," Toxic Effects of Mercury, Springer, Berlin, Germany, 2014.

[36] G. R. Inmaculada, R. J. Cristina, D. S. Teresa et al., "Uric acid and anti-TNF antibody improve mitochondrial dysfunction in ob/ob mice," Hepatology, vol. 44, pp. 581-591, 2006.

[37] S. J. Burke, D. Lu, T. E. Sparer, M. D. Karlstad, and J. J. Collier, "Transcription of the gene encoding TNF- $\alpha$ is increased by IL$1 \beta$ in rat and human islets and $\beta$-cell lines," Molecular Immunology, vol. 62, no. 1, pp. 54-62, 2014.

[38] C. Liu, X. Feng, Q. Li, Y. Wang, Q. Li, and M. Hua, "Adiponectin, TNF- $\alpha$ and inflammatory cytokines and risk of type 2 diabetes: a systematic review and meta-analysis," Cytokine, vol. 86, pp. 100-109, 2016.

[39] H. Heimberg, Y. Heremans, C. Jobin et al., "Inhibition of cytokine-induced NF- $\kappa \mathrm{B}$ activation by adenovirus-mediated expression of a NF- $\kappa \mathrm{B}$ super-repressor prevents $\beta$-cell apoptosis," Diabetes, vol. 50, no. 10, pp. 2219-2224, 2001.

[40] E. Brioudes, M. Alibashe-Ahmed, V. Lavallard, T. Berney, and D. Bosco, "Syndecan-4 is regulated by IL-1beta in beta-cells and human islets," Molecular and Cellular Endocrinology, vol. 510, Article ID 110815, 2020.

[41] A. Akrami, F. Nikaein, S. Babajafari, S. Faghih, and H. Yarmohammadi, "Comparison of the effects of flaxseed oil and sunflower seed oil consumption on serum glucose, lipid profile, blood pressure, and lipid peroxidation in patients with metabolic syndrome," Journal of Clinical Lipidology, vol. 12, no. 1, pp. 70-77, 2018.

[42] E. K. Vassiliou, A. Gonzalez, C. Garcia et al., "Oleic acid and peanut oil high in oleic acid reverse the inhibitory effect of insulin production of the inflammatory cytokine TNF-alpha both in vitro and in vivo systems," Lipids in Health and Disease, vol. 8, no. 25, pp. 1-10, 2009.

[43] C.-C. Chen, Y.-C. Lu, Y.-W. Chen et al., "Hemopexin is upregulated in plasma from type 1 diabetes mellitus patients: role of glucose-induced ROS," Journal of Proteomics, vol. 75, no. 12, pp. 3760-3777, 2012. 
[44] A. M. Al-Attar, "Therapeutic influences of almond oil on male rats exposed to a sublethal concentration of lead," Saudi Journal of Biological Sciences, vol. 27, no. 2, pp. 581-587, 2020.

[45] Y. Gao, J. T. Li, S. F. Chu et al., "Ginsenoside Rg1 protects mice against streptozotocin-induced type 1 diabetic by modulating the NLRP3 and Keap1/Nrf2/HO-1 pathways," European Journal of Pharmacology, vol. 866, Article ID 172801, 2020.

[46] C. A. Agca, M. Tuzcu, A. Hayirli, and K. Sahin, "Taurine ameliorates neuropathy via regulating NF- $\kappa \mathrm{B}$ and $\mathrm{Nrf} 2 / \mathrm{HO}-1$ signaling cascades in diabetic rats," Food and Chemical Toxicology, vol. 71, pp. 116-121, 2014.

[47] H. Han, Y. Y. Li, J. Fang et al., "Gut microbiota and type 1 diabetes," International Journal of Molecular Sciences, vol. 19, no. $4,2018$.

[48] J. Z. Wu and L. J. Yan, "Streptozotocin-induced type 1 diabetes in rodents as a model for studying mitochondrial mechanisms of diabetic beta cell glucotoxicity," Diabetes, Metabolic Syndrome and Obesity: Targets and Therapy, vol. 8, pp. 181-188, 2015.

[49] M. Lyu, Y. F. Wang, G. W. Fan et al., "Balancing herbal medicine and functional food for prevention and treatment of cardiometabolic diseases through modulating gut microbiota," Frontiers in Microbiology, vol. 8, Article ID 02146, 2017.

[50] C. P. Tamboli, C. Neut, P. Desreumaux, and J. F. Colombel, "Dysbiosis in inflammatory bowel disease," Gut, vol. 53, no. 1, pp. 1-4, 2004.

[51] L. Wang, Q. Y. Shang, W. X. Guo et al., "Evaluation of the hypoglycemic effect of probiotics via directly consuming glucose in intestines of STZ-induced diabetic mice and glucose water-induced diabetic mice," Journal of Functional Foods, vol. 64, Article ID 103614, 2020.

[52] R. Zhao, Q. Hu, G. Ma et al., "Effects of flammulina velutipes polysaccharide on immune response and intestinal microbiota in mice," Journal of Functional Foods, vol. 56, pp. 255-264, 2019.

[53] A. E. Yanni, G. Mitropoulou, I. Prapa et al., "Functional modulation of gut microbiota in diabetic rats following dietary intervention with pistachio nuts (Pistacia vera L.)," Metabolism Open, vol. 7, Article ID 100040, 2020.

[54] D. N. Frank, A. L. Amand, R. A. Feldman, E. C. Boedeker, N. Harpaz, and N. R. Pace, "Molecular-phylogenetic characterization of microbial community imbalances in human inflammatory bowel diseases," Proceedings of the National Academy of Sciences, vol. 104, no. 34, pp. 13780-13785, 2007.

[55] Q. T. Ma, Y. Q. Li, J. K. Wang et al., "Investigation of gut microbiome changes in type 1 diabetic mellitus rats based on high-throughput sequencing," Biomedecine \& Pharmacotherapie, vol. 124, Article ID 109873, 2020.

[56] W. T. Zhou, G. J. Chen, D. Chen, H. Ye, and X. X. Zeng, "The antidiabetic effect and potential mechanisms of natural polysaccharides based on the regulation of gut microbiota," Journal of Functional Foods, vol. 75, Article ID 104222, 2020.

[57] Y. Li, Q. Ma, J. Wang et al., "Relationship between hyperlipidemia and the gut microbiome of rats, characterized using high-throughput sequencing," Journal of Traditional Chinese Medical Sciences, vol. 7, no. 2, pp. 154-161, 2020.

[58] H. X. Cui, Y. N. Hu, J. W. Li, and K. Yuan, "Hypoglycemic mechanism of the berberine organic acid salt under the synergistic effect of intestinal flora and oxidative stress," Oxidative Medicine and Cellular Longevity, vol. 2018, Article ID 8930374, 13 pages, 2018.
[59] M. L. Xue, Y. Liu, H. W. Xu et al., "Propolis modulates the gut microbiota and improves the intestinal mucosal barrier function in diabetic rats," Biomedicine \& Pharmacotherapy, vol. 118, Article ID 109393, 2019.

[60] Q. Z. Ding, B. W. Zhang, W. Zhang et al., "Liupao tea extract alleviates diabetes mellitus and modulates gut microbiota in rats induced by streptozotocin and high-fat, high-sugar diet," Biomedicine \& Pharmacotherapy, vol. 118, Article ID 109262, 2019.

[61] S. Hu, J. Wang, J. Wang, H. Yang, X. Yan, and L. Su, "Fucoidan from acaudina molpadioides improves insulin resistance by altering gut microbiota dysfunction," Journal of Functional Foods, vol. 57, pp. 59-67, 2019.

[62] M. Gurung, Z. P. Li, H. You et al., "Role of gut microbiota in type 2 diabetes pathophysiology," EBioMedicine, vol. 51, Article ID 102590, 2020.

[63] B. Tian, M. Liu, W. An et al., "Lycium barbarum relieves gut microbiota dysbiosis and improves colonic barrier function in mice following antibiotic perturbation," Journal of Functional Foods, vol. 71, Article ID 103973, 2020. 